\title{
Schon moderate Aktivität senkt Mortalität
}

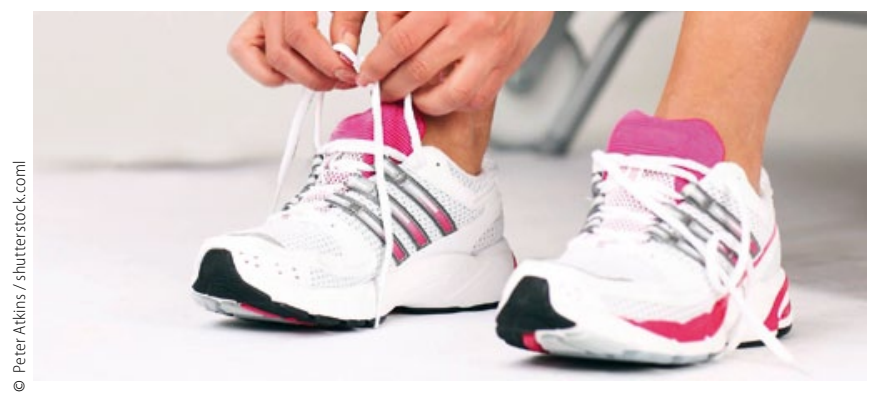

Aufgeht's! Turnschuhe an und eine Runde um den Block - schon ein wenig Bewegung im Alltag lässt Diabetiker länger leben.

Fragestellung: Wie intensiv muss sich ein Diabetiker in Beruf und Freizeit körperlich betätigen bzw. welche Strecken muss er gehen, um sein Leben zu verlängern?

Hintergrund: Körperliche Aktivität ist sowohl für gesunde Menschen als auch für Diabetiker nützlich, denn sie verlängert das Leben und schützt vor kardiovaskulären Erkrankungen. Unzureichend sind die Informationen über die Dosis-Wirkungs-Kurve, also welches Ausmaß an Aktivität erforderlich ist, um einen messbaren Nutzen zu gewährleisten.

Patienten und Methodik: Die Daten stammen aus der European Prospective Investigation into Cancer and Nutrition (EPIC-)Studie, an der sich 23 Zentren in 10 europäischen Ländern, darunter mehrere in Deutschland, beteiligen. Unter den 519978 Probanden im Alter

Sluik D, Buijsse B, Muckelbauer R et al.: Physical activity and mortality in individuals with diabetes mellitus. A prospective study and meta-analysis. Arch. Intern. Med.2012;172(17)1285-95. zwischen 35 und 70 Jahren befanden sich 5859 Diabetiker. Die gesamte körperliche Aktivität wurde aus beruflicher Beanspruchung plus Gehen und Radfahren bestimmt, die Freizeitaktivität aus Gehstrecke,
Radfahren, Gartenarbeit, Sport, Haushaltsarbeit und handwerklicher Betätigung. Die Einteilung erfolgte jeweils in vier Aktivitätskategorien. Bei der Auswertung der Ergebnisse wurden viele unterschiedliche Einflussfaktoren berücksichtigt.

Ergebnisse: Während der Studiendauer von 9,4 Jahren starben 755 Probanden, darunter 212 infolge kardiovaskulärer Ursachen. Im Vergleich zur Gruppe der am wenigsten aktiven Diabetiker nahmen Gesamtmortalität und kardiovaskuläre Mortalität schon bei den gering aktiven in den Kategorien gesamte körperliche Aktivität, Freizeitaktivität und 2-4,5 Stunden (statt weniger als 2 Stunden) Gehen pro Woche um 13-32 bzw. 9-60\% ab (Tab. 1). Häufig wurde der Hauptnutzen schon in der gering aktiven Gruppe erreicht. Manchmal meint man zu erkennen, dass mit vermehrter Aktivität der Nutzen linear zunimmt.

Schlussfolgerung: Bei Diabetikern führt schon moderate körperliche Aktivität in Beruf und Freizeit zu einem deutlichen Rückgang der Gesamtsterblichkeit und der kardiovaskulären Mortalität.

\begin{tabular}{|c|c|c|c|c|}
\hline & Inaktiv & Wenig aktiv & mäßig aktiv & Sehr aktiv \\
\hline & \multicolumn{4}{|c|}{ Gesamte körperliche Aktivität } \\
\hline Ges-Mortalität & 1 & $-32 \%$ & $-42 \%$ & $-19 \%$ \\
\hline \multirow[t]{2}{*}{ CV-Mortalität } & 1 & $-54 \%$ & $-69 \%$ & $-52 \%$ \\
\hline & \multicolumn{4}{|c|}{ Freizeit-Aktivität } \\
\hline Ges-Mortalität & 1 & $-23 \%$ & $--21 \%$ & $-38 \%$ \\
\hline \multirow[t]{2}{*}{ CV-Mortalität } & 1 & $-9 \%$ & $-31 \%$ & $-70 \%$ \\
\hline & \multicolumn{4}{|c|}{ Gehen Std./Woche } \\
\hline Ges-Mortalität & 1 & $-13 \%$ & $-24 \%$ & $-10 \%$ \\
\hline CV-Mortalität & 1 & $-60 \%$ & $-56 \%$ & $-35 \%$ \\
\hline
\end{tabular}

\section{-Kommentar von Prof. Dr. med. Heinrich Holzgreve}

\section{Mit 2500 bis 6000 Schritte pro Tag das Leben verlängern}

Als einer der ganz wichtigen Maßnahmen für Diabetiker wird die körperliche Aktivität empfohlen. In den Leitlinien reicht das Spektrum der "Dosis"-Empfehlungen in der Regel von „mindestens $21 \frac{1}{2}$ Stunden pro Woche“ bis "so viel körperliche Betätigung wie möglich". Tatsächlich ist schon die Minimal-Forderung sehr erfolgreich.

Eine der besonders wichtigen Erkenntnisse dieser Studie betrifft das Gehen, für das man kein Fitnessstudio, keinen Sportplatz und keine Geräte benötigt. Schon eine Dauer von 2-4,5 Stunden pro Woche - etwa 2500 bis 6000 Schritte pro Tag bringen einen erheblichen Nutzen. Die Autoren legen gleichzeitig mit ihren Daten eine Metaanalyse von 12 Studien zur körperlichen Aktivität bei Diabetes mellitus mit qualitativ und quantitativ ähnlichen Ergebnissen vor.

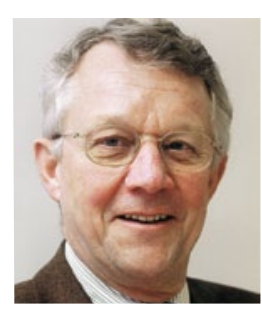

Prof. Dr. med. Heinrich Holzgreve

Internist, Kardiologische Praxis

Burgstr. 7, 80331 München

h.holzgreve@t-online.de 